\title{
Amyloidosis of the spine in a patient on long-term hemodialysis
}

\author{
L. A. Welk and D.J. Quint \\ Department of Neuroradiology, University of Michigan Hospitals, Ann Arbor, Michigan, USA
}

Received: 13 October 1989

\begin{abstract}
Summary. A 54-year-old man with a history of renal failure treated with hemodialysis for over 10 years presented with clinical signs and symptoms and plain radiographic, computed tomographic and magnetic resonance imaging features of cervical vertebral osteomyelitis with spinal cord compression. Decompressive surgery revealed amyloid deposition. In the setting of chronic hemodialysis, differentation between amyloid deposition and osteomyelitis may not be possible on an imaging basis necessitating biopsy for diagnosis.
\end{abstract}

Key words: MR - Spine - Infection - Amyloid - Hemodialysis

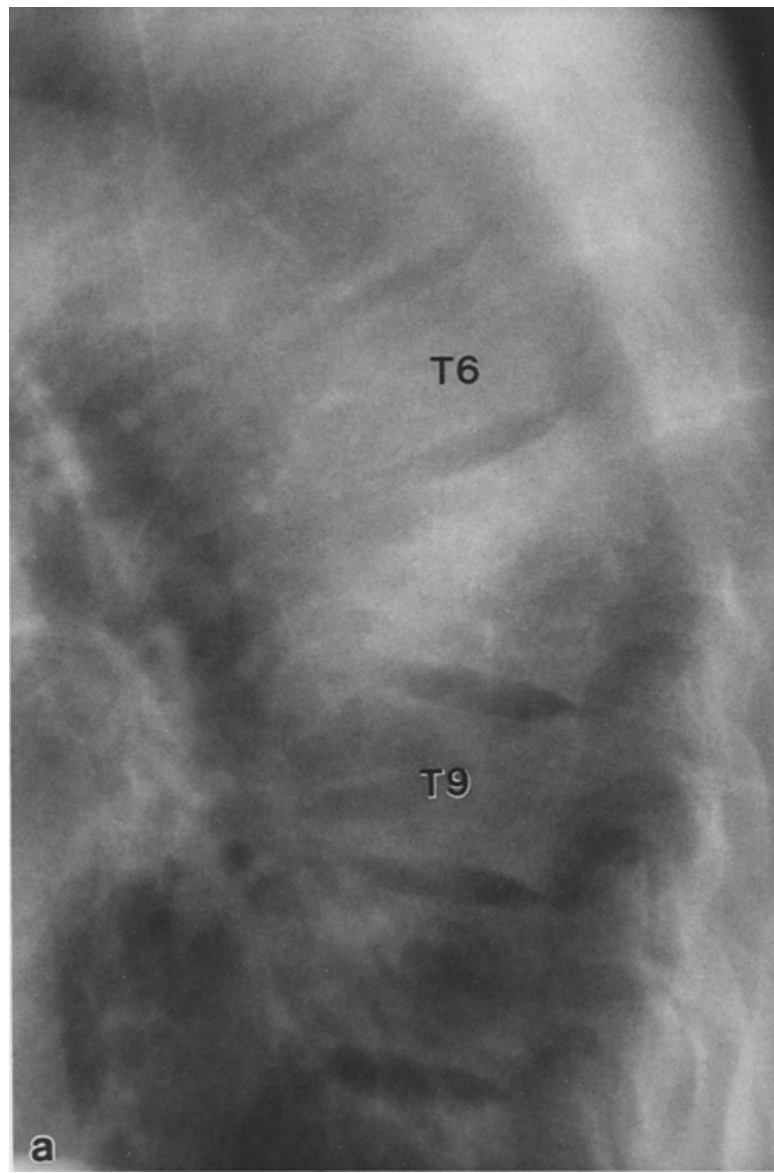

When lesions suggestive of destructive spondyloarthropathy occur in patients undergoing long-term hemodialysis, possible etiologies include infection, secondary hyperparathyroidism, crystal deposition, and neuropathic change. We report a case where such a lesion was caused by amyloid deposition with imaging characteristics indistinguishable from infection. Similar cases have been described by Sebert et al. [1], Orzincolo et al. [2], and Naidich et al. [3]. The plain radiographic, computed tomographic, and magnetic resonance imaging characteristics of this lesion are described.

\section{Case report}

A 54-year-old man with end-stage renal disease secondary to hypertension who had been treated by hemodialysis for over ten years presented with a 2 month history of grad-

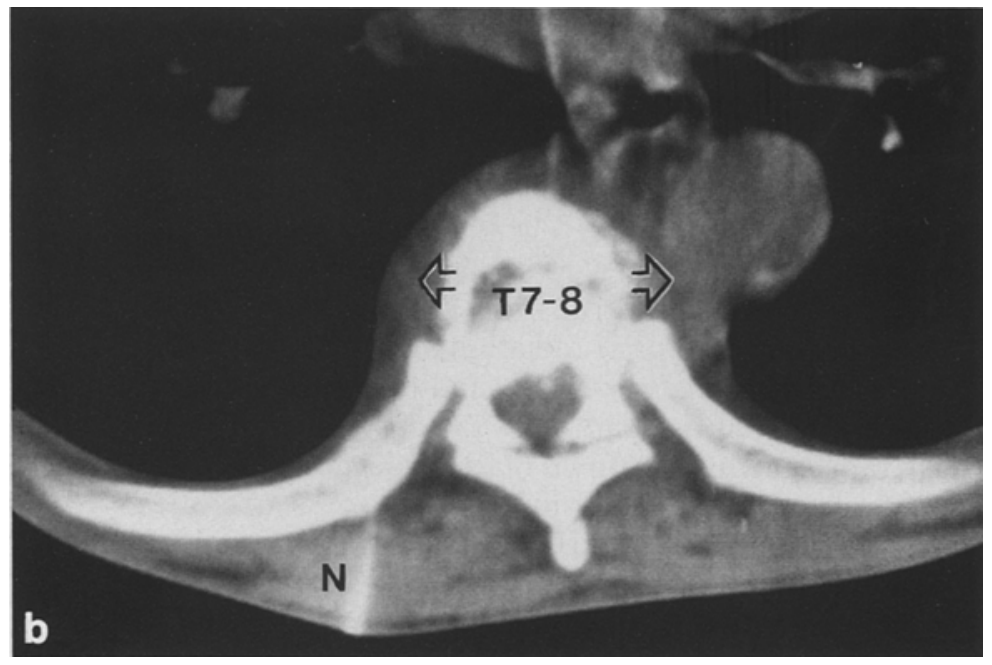

Fig.1a,b. Suspected, but not definitely proven amyloidosis of the mid-thoracic spine. Lateral thoracic radiograph (a) demonstrates loss of the T7-8 intervertebral disc space with endplate destruction and vertebral body compression involving predominantly the superior portion of $\mathrm{T} 8$, but also the inferior portion of T7. Noncontrast CT at this level (b) demonstrates bilateral paraspinal extension of abnormal soft tissue (arrows). Findings are most consistent with infection. However, biopsy ( $N=$ needle) was culture-negative. Special stains (congo red, crystal violet) were suspicious (see text) for amyloid 

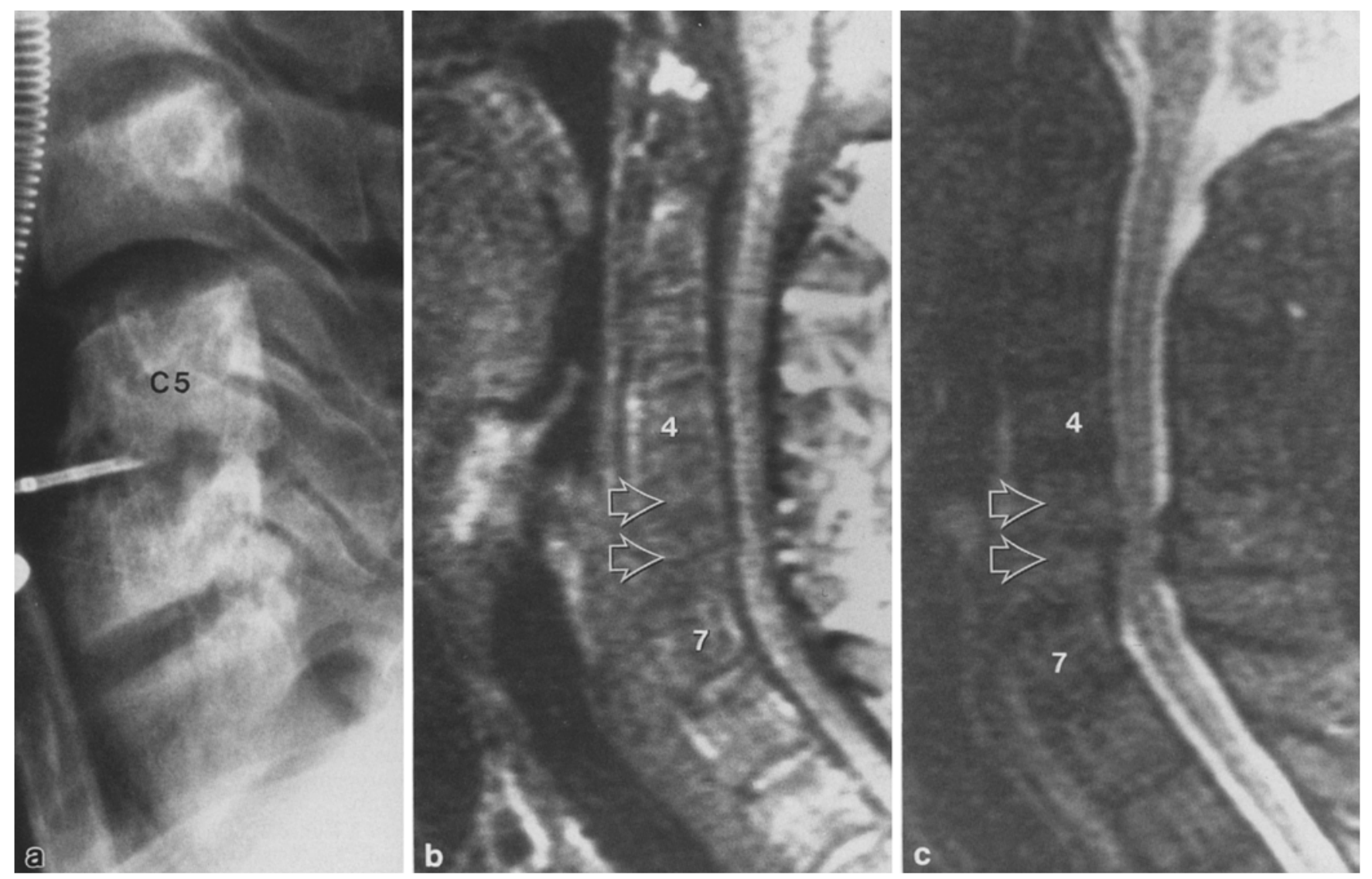

Fig. 2a-c. Biopsy proven amyloid of the cervical spine. Lateral cervical spine radiograph (a) demonstrates endplate destruction, intervertebral disc space narrowing and minimal retrolisthesis at C5-6. Sagittal T1-weighted (TR/TE $=600 / 25$ ) MR (b) demonstrates decreased signal from the $\mathrm{C} 5$ and $\mathrm{C} 6$ vertebral bodies and the intervening intervertebral disc (arrows). Sagittal T2-weighted $(\mathrm{TR} / \mathrm{TE}=2000 / 80) \mathrm{MR}(\mathrm{c})$ demonstrates subtle increased signal from these vertebrae and the C5-6 disc (arrows). Spinal cord compression is also seen at this level. The plain film findings are most suggestive of infection and while somewhat atypical, the MR findings are also consistent with infection. Surgical decompression with biopsy was culture negative; stains were positive for amyloid

ually increasing interscapular pain which radiated to the left shoulder. He also complained of localized pain and tenderness over the spine. The pain had been initially relieved with non-steroidal antiinflammatory medication, but subsequently became persistent with episodes lasting up to nine hours per day. The patient denied fever, chills, sweats, or a history of trauma. The patient did report a 10 pound weight loss over the preceding 10 months which he attributed to a decreased appetite. Physical exam revealed tenderness over the thoracic spine. No focal neurologic deficits were identified. Spinal radiographs demonstrated loss of intervertebral disc space with associated end-plate destruction at the T7-8 (Fig. 1a) and C5-C6 (Fig. 2a) levels. The Westergren Sedimentation Rate (WESR) at the time of admission was 115 . Other lab tests were unremarkable.

A non-contrast computed tomographic examination of the thoracic spine (Fig. 1b) demonstrated partial de- struction of the $\mathrm{T} 7$ and $\mathrm{T} 8$ vertebral bodies by abnormal soft tissue that extended into the paraspinal region bilaterally obliterating the normal paraspinal mediastinal fat planes. A CT-guided biopsy of this mass was performed and samples were sent for culture as well as for congo red and crystal violet staining. No organisms were identified on the microbiology studies. The studies for amyloid were suggestive of amyloidosis, but ultimately were deemed inconclusive secondary to an inadequate amount of specimen.

An MR examination (GE Signa, 1.5 Tesla) of the cervical spine revealed spinal stenosis from $\mathrm{C} 5$ to $\mathrm{C} 7$ with the suggestion of spinal cord compression due to abnormal soft tissue located both anterior and posterior to the thecal sac. On the short TR, short TE (T1-weighted) scan (Fig. 2b), decreased signal from the C5 and C6 vertebral bodies as well as in the expected location of the C5-C6 intervertebral disk was seen. On the long TR, long TE (T2weighted) scan (Fig. 2c), slightly increased signal was seen in this region. Infection could not be excluded and the patient underwent an open biopsy (surgical decompression) of the cervical lesion. Congo red and crystal violet stains were postive for amyloid.

\section{Discussion}

Patients undergoing long-term hemodialysis suffer from various problems of the bones and joints including renal osteodystrophy, infections, and acute arthritis or inflam- 
mation of the soft tissues adjacent to joints, which in some cases may be related to crystal deposition. More recently it has become apparent that these patients may also suffer from a destructive spondyloarthropathy. Some authors have attributed this spondyloarthropathy to crystal deposition [4] while others have shown that such lesions may be secondary to amyloid deposition as appears to be the case in our patient.

Amyloid deposits can be found in the hands and wrists of dialysis patients and are associated with an osteoarthropathy and carpal tunnel syndrome. The presence of amyloid deposition at one anatomic site increases the probability of its presence at other sites [3]. The risk of developing such disease is related to the duration of dialysis. Biochemically, amyloid deposits in this syndrome consist of B-2 microglobulin, a protein that accumulates in uremic patients, especially those patients undergoing hemodialysis with units that utilize cuprophan membranes. This protein appears to play a major role in the pathogenesis of amyloid deposition [5].

The basic clinical problem in symptomatic hemodialysis patients who present with destructive vertebral lesions is to rule out osteomyelitis. Osteomyelitis usually demonstrates decreased signal intensity on T1-weighted sequences and increased signal intensity on T2-weighted sequences [6]. In the spine, in the appropriate clinical setting, when such findings involve a disc and adjacent vertebrae, infection should be suspected. However, it should be noted that there have been reports of culture-proven osteomyelitis that demonstrate decreased signal intensity on both the T1-weighted and T2-weighted sequences [7]. Therefore, while not entirely typical, the observed findings on the MR study in our patient are not inconsistent with infection. Although our patient was afebrile, he did present with localized complaints of pain and tenderness over the spine as well as an elevated sedimentation rate. Therefore, in this case, it was impossible to exclude infection on the basis of the history, physical exam, plain radiographic, computed tomographic, or magnetic resonance imaging findings all of which were consistent with infec- tion. The patient ultimately required an open biopsy for diagnosis.

Amyloid deposition should be considered a potential cause of destructive spondyloarthropathy in patients undergoing long-term hemodialysis. The presence of osteoarthropathy in the hands and wrists (with or without associated carpal tunnel syndrome) should further increase clinical suspicion of amyloid deposition as the causative agent. Plain film, computed tomographic, and magnetic resonance imaging findings appear to be nonspecific and may simulate infection in these patients. Biopsy may be required for definitive diagnosis.

\section{References}

1. Sebert JL, Fardellone P, Deramond H, Marie A, Lansaman J, Bardin T, Lambrey G, Gheerbrant JD, Legars D, Galibert P (1986) Spondylarthropathie destructive avec depots amyloides chez 3 patients en hemodialyse chronique. Rev Rhum 53:459-465

2. Orzincolo C, Bedani PL, Scutellari PN, Cardona P, Farinelli A, Vita G (1988) L amiloidosi osteoarticolare da dialisis. Radiol Med 76: $38-43$

3. Naidich JB, Mossey RT, McHeffey-Atkinson B, Karmel MI, Bluestone PA, Mailloux LU, Stein HL (1988) Spondyloarthropathy from long-term hemodialysis. Radiology 167: 761-764

4. Kuntz D, Naveau B, Bardin T, Drueke T, Treves R, Dryll A (1984) Destructive spondyloarthropathy in hemodialyzed patients. Arthritis Rheum 27:369-375

5. Bardin T, Zingraff J, Benoit J, Kuntz D, Drueke T (1987) L amylose des hemodialyses. Press Med 16:907-910

6. Unger E, Moldofsky P, Gatenby R, Hartz W, Broder G (1988) Diagnosis of osteomyelitis by MR imaging. AJR 150: 605-610

7. Modic MR, Pflanze W, Feiglin DHI, Belhobek G (1986) Magnetic resonance imaging of musculoskeletal infections. Radiol Clin North Am 24: 247-267

\section{D.J.Quint, M.D.}

Department of Neuroradiology

Room B1D530H, Box 0030

University of Michigan Hospitals

1500 East Medical Center Drive

Ann Arbor, MI 48109-0030

USA 